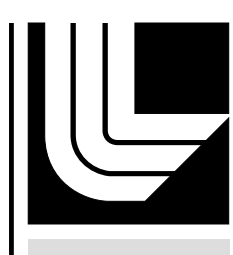

LAWRENCE LIVERMORE N A T IO N A L LABORATORY

\title{
Developing Models for the DIII-D Boundary Plasma
}

G. D. Porter, T. D. Rognlien, M. E. Rensink, P. C. Stangeby, N. S. Wolf

May 25, 2005

Fusion Science 
This document was prepared as an account of work sponsored by an agency of the United States Government. Neither the United States Government nor the University of California nor any of their employees, makes any warranty, express or implied, or assumes any legal liability or responsibility for the accuracy, completeness, or usefulness of any information, apparatus, product, or process disclosed, or represents that its use would not infringe privately owned rights. Reference herein to any specific commercial product, process, or service by trade name, trademark, manufacturer, or otherwise, does not necessarily constitute or imply its endorsement, recommendation, or favoring by the United States Government or the University of California. The views and opinions of authors expressed herein do not necessarily state or reflect those of the United States Government or the University of California, and shall not be used for advertising or product endorsement purposes. 


\title{
Developing models for the DIII-D boundary plasma
}

G.D. Porter, T.D. Rognlien, M.E. Rensink, P.C. Stangeby,* and N.S. Wolf

Lawrence Livermore National Laboratory, Livermore, California

*University of Toronto Institute for Aerospace Studies, Toronto, Canada.

Contact author: G.D. Porter, Lawrence Livermore National Laboratory, P.O. Box 808 (L-637), Livermore, California 94551-9900, Phone (925) 423-9391, Fax (925) 423-6401, e-mail: porter2@1lnl.gov

Total pages: 48 (36 text, 11 figures, 1 table)

(Received

\begin{abstract}
Development of the comprehensive codes used to study the boundary region of the DIII-D tokamak has been done in parallel with improvement of the diagnostics of this important region of the plasma. These codes have been used to interpret the diagnostic data and assist in design of improved divertor configurations. The development of codes used for analysis on DIII-D is described briefly. Model validation by comparing with the extensive DIII-D boundary region diagnostic data is also discussed.
\end{abstract}

PACs Nos. $52.65 . \mathrm{Kj}, 52.55 . \mathrm{Rk}, 52.55 . \mathrm{Fa}$ 


\section{INTRODUCTION}

The boundary region of a tokamak plasma is important for at least two reasons. First, it encompasses the edge confinement barrier seen in high confinement modes (H-mode) and is therefore important in determining the global confinement properties of the confined plasma, and secondly, the boundary region includes the scrape-off-layer plasma which determines the interaction with material walls. Examination of the physics of the boundary region of a diverted tokamak plasma is made difficult by the fact the relevant physics is inherently $2-\mathrm{D}$, perhaps even $3-\mathrm{D}$. The boundary region is defined to be that portion of the plasma which extends from a closed flux surface near the separatrix (approximately the $90 \%$ to the $95 \%$ poloidal flux surface) radially outward to the plasma facing material surfaces. The physics issues which are important in this region of a tokamak has been discussed in detail by Stangeby [1]. The flux surfaces in the boundary region of a typical DIII-D double-null plasma are shown in Fig. 1. Understanding the physics of this plasma region necessarily combines the issues of the H-mode pedestal region on the closed surfaces, and those associated with the open field lines of the scrapeoff layer (SOL). The interaction of the plasma in the SOL with material surfaces leads to both recycling of fuel ions as neutrals, and generation of impurities via sputtering. These interactions are localized to regions where the plasma interacts with the material. Ideally, these interaction regions are toroidally symmetric, arising only where the open field lines of the SOL intersect the divertor plates. These interactions then require a 2D code to model plasma transport in the boundary region. Unfortunately, the SOL plasma may also interact with localized regions which are not toroidally symmetric, e.g. bumper limiters. If these interactions are important, a 3D model would be required to model the boundary plasma. Furthermore, a complete description of plasma turbulence does require a 3D model. Since it is impractical to obtain a complete diagnosis of the boundary plasma, the 
physics of the region must be examined by combining the best available diagnostic information with comprehensive models which incorporate the best available physics understanding.

The requirement for comprehensive models was recognized as an important component required for successful design of a divertor concept for proposed next generation experiments including the International Thermonuclear Experimental Reactor (ITER) and Fusion Ignition Research Experiment (FIRE). Since the divertor was perceived to be a component whose failure would prevent ITER from achieving its goals, there was a worldwide program initiated to develop and validate the requisite comprehensive models. This code development program has been described previously [2], and we will rely rather heavily on the work in that publication. The worldwide code development led to the creation of three main 2D models, B2/EIRENE used primarily in Europe, EDGE2D/NIMBUS used primarily at the JET facility, and UEDGE used primarily on DIII-D. In addition to these $2 \mathrm{D}$ codes, effort has been expended to develop a simpler interpretive code which essentially solves the fluid equations separately in each flux tube, and is therefore 1D numerically, although producing 2D solutions. Radial effects are approximated by solving the 1D equations on adjoining flux tubes. This code Onion-Skin Modeling plus EIRENE and DIVIMP for edge interpretive analysis (OEDGE) has been applied to many of the present tokamak devices, including DIII-D [3].

There has been significant efforts to apply each of these codes to multiple devices to ensure the viability of the models used in each code. Since the UEDGE and OEDGE codes are the primary tools used on the DIII-D device, this report will focus on the development of these codes. Other codes have been used for analysis of DIII-D boundary plasmas [4-12]. Space limitations, however, prevent detailed discussion of all code results, so we have chosen to focus on the results of the codes which are most frequently 
used. We start with a discussion of the basic equations used in boundary physics codes in Section II. We follow with a description of both the UEDGE and OEDGE codes in Sections III and IV. An important component of the development of successful modeling codes is validation of the codes against the most comprehensive diagnostic data available. We describe the results of these code validation studies on DIII-D in Sections V and VI. Finally, we conclude with a summary in Section VII. 


\section{BASIC EQUATIONS}

This description of the basic equations of the comprehensive models used to study the physics of the boundary plasma in DIII-D follows very closely the description used in the ITER physics basis [2]. We have modified that description to focus on the models applied to DIII-D. A realistic description of the tokamak boundary plasma requires models for the transport of both plasma particles and neutral gas, the line radiation emitted during electron-ion and electron-neutral collisions, and the recombination of plasma to neutrals. The neutrals include both the fuel hydrogen species and various impurities, either intrinsic or those injected to control the divertor plasma. All of these species are tightly coupled, and their effects must be included self-consistently.

\section{A. Plasma transport}

The boundary plasma transport equations used in the codes applied in the DIII-D program are solved in the poloidal plane assuming uniformity in the toroidal direction. The coordinates used are usually orthogonal, i.e. poloidal (x, along the poloidal projection of the field lines) and radial (y, perpendicular to this projection), except in regions near the divertor plates where the mesh is distorted to fit the shape of the plates. The flux surface mesh is obtained from the magnetohydrodynamic (MHD) equilibrium reconstruction obtained using EFIT [13]. Poloidal and radial coordinates are chosen to separate parallel and radial transport, with different time scales. The transport equations can be cast in the form of convection/diffusion equations:

$$
\frac{\partial \psi_{\mathrm{j}}}{\partial \mathrm{t}}+\nabla \cdot\left(\mathbf{v}_{\mathrm{j}} \psi_{\mathrm{j}}-\mathbf{D} \cdot \nabla \psi_{\mathrm{j}} / n_{\mathrm{j}}\right)=S_{\psi \mathrm{j}}
$$

Here $\psi_{\mathrm{j}}$ represents the plasma variables: $n_{\mathrm{j}}, m_{j} n_{\mathrm{j}} \mathbf{v}_{\mathrm{pj}}, n_{\mathrm{e}} T_{\mathrm{e}}$, or $n_{\mathrm{i}} T_{\mathrm{i}}$, where $n_{\mathrm{j}}, m_{\mathrm{j}}$, and $\mathrm{v}_{\mathrm{pj}}$ are the density, mass and parallel velocity of ion species $\mathrm{j}: n_{\mathrm{e}}=\sum n_{\mathrm{j}} Z_{\mathrm{j}}$, and $T_{\mathrm{e}}$ are 
the electron density and temperature, $Z_{\mathrm{j}}$ is the ion charge state, and $n_{\mathrm{i}} T_{\mathrm{i}}=\sum n_{\mathrm{j}} T_{\mathrm{j}}$ is the total ion energy density. Also, $\mathbf{v}_{\mathrm{j}}$ is the ion velocity vector, and $\mathbf{D}$ is a diffusion tensor. The strong source terms, $S_{\psi \mathrm{j}}$, are due to collisions of the plasma with neutral atoms and molecules.

The UEDGE code $[14,15]$ uses a fully implicit approach in which all the equations are solved simultaneously using a Newton-Krylov method [16]. This approach can be used to find steady state solutions directly or to follow transients.

Poloidal transport is taken as the geometrical projection of the classical parallel transport from Braginskii [17] for hydrogenic species. The impurity parallel transport is also classical, including the effects of the thermal forces and inter-species friction [18]. Flux limits are applied to the transport terms such as viscosity and thermal conductivity, to prevent unphysically large values when the mean free path of the plasma particles becomes longer than gradient scale lengths [19-21].

Radial transport is described by anomalous diffusion and convection velocity coefficients, chosen to be consistent with experimental profiles at various locations (midplane, divertor). The uncertainty in the nature of radial transport is one of the key difficulties in permitting prediction with the present simulation codes. An estimate of the effective radial transport coefficients can be made for any discharge by fitting the measured density and temperature profile. However, it is difficult to determine how these coefficients scale, hence it is difficult to predict the behavior of the boundary plasma in future experiments or devices. Recently, there has been significant effort in determining radial transport rates from first principles, using 3D turbulence simulations with the BOUT code [22-24]. This turbulence code is now being coupled with the UEDGE code to permit comprehensive simulation of all the physics, using cross-field transport determined from first principles $[25,26]$. The effects of parallel current are incorporated 
in many of the simulations described in this paper. This changes some details of the solution such as producing larger in/out power asymmetry for a single-null divertor, resulting in higher power to the outer plate and easier detachment at the inner plate. The effect of classical cross field drifts have been included in recent simulations, and are found to be significant [27]. The source terms in the plasma transport equations arise from ionization, recombination, and charge exchange collisions between plasma and neutral species. For example, in the ion continuity equations, the source term, $S_{\mathrm{n}, \mathrm{j}}$, for the impurity ions, takes on the form

$$
\begin{aligned}
S_{\mathrm{n}, \mathrm{j}}= & n_{\mathrm{e}}\left(n_{\mathrm{j}-1} K_{\mathrm{j}-1}^{\mathrm{ion}}+n_{\mathrm{j}+1} K_{\mathrm{j}+1}^{\mathrm{rec}}\right)-n_{\mathrm{e}} n_{\mathrm{j}}\left(K_{\mathrm{j}}^{\mathrm{ion}}+K_{\mathrm{j}}^{\mathrm{rec}}\right) \\
& +n_{\mathrm{H}}\left(n_{\mathrm{j}+1} K_{\mathrm{j}+1}^{\mathrm{cx}}-n_{\mathrm{j}} K_{\mathrm{j}}^{\mathrm{cx}}\right) \ldots
\end{aligned}
$$

Here, $n_{\mathrm{H}}$ is the neutral hydrogen density. The rate coefficients for ionization, $K^{\text {ion }}$, charge exchange $K^{\mathrm{cx}}$ and recombination $K^{\mathrm{rec}}$, and the associated energy exchange for the latter two are taken from various databases. UEDGE uses hydrogen rates from Refs. [28] and [29], and impurity rates from STRAHL [30] or ADAS [31]. OEDGE uses hydrogen rates from EIRENE or ADAS, and impurity rates from ADAS, STRAHL, or ADPACK.

\section{B. Neutral transport}

Neutral transport in the boundary codes used on DIII-D is treated either by Monte Carlo techniques, or by a reduced Navier-Stokes fluid model. Although some simulations

done using the UEDGE code incorporate the Monte Carlo techniques [32], most have been done using the Navier-Stokes model [33]. The OEDGE codes incorporate the Monte Carlo technique, using the EIRENE code [34]. 


\section{Neutral Fluid Model}

The Navier-Stokes fluid model used in UEDGE [33] solves a parallel momentum equation for the neutral velocity, but treats the motion across $\mathrm{B}$ as diffusive (dominated by charge exchange collisions). This model yields results similar to those of a neutral model that solves for all three momentum components [35]. The viscosity and thermal conduction coefficients for the neutrals account for both charge exchange and neutralneutral collisions. The ionization and recombination terms in Eq. (2) are handled directly. Neutral energy transport is treated with a combined ion/neutral energy equation. Neutrals are recycled at the walls as atoms using tabulated surface reflection coefficients so that a separate equation for molecules is not used. However, the electron energy loss and ion energy gains due to molecular dissociation are accounted for by removing the energy for dissociation (about $10 \mathrm{eV}$ ) from the electrons upon ionization.

The neutral flux is limited to its thermal free streaming value to prevent excessive transport in low plasma density regions. The fluid neutral equations are treated simultaneously with the plasma equations so that the whole system is advanced in a numerically implicit manner, allowing large time steps, thus permitting the study of slowly varying phenomena.

\section{Monte Carlo model}

The Monte Carlo neutral transport codes solve a kinetic equation for the neutral particle distribution function. The plasma source (or sink) terms, e.g. Eq. (2) computed as averages of this distribution, are coupled back to the plasma code (assuming quasisteady-state neutrals). Long neutral trajectories in low plasma density regions are accurately computed using this method, but it is costly in regions in which charge 
exchange collisions are dominant and neutral transport is mostly diffusive. Both atoms and molecules are treated.

The Monte Carlo codes treat in detail the plasma-surface interactions: reflection, adsorption and thermal emission, physical sputtering, and chemical erosion (see reviews $[36,37])$. 


\section{2D MODEL (UEDGE)}

The UEDGE code, developed by various groups $[14,15]$ originated from an early version of the B2 code [38] modified to be fully implicit. Early versions of the code incorporated a very simple neutral model, and were restricted to an orthogonal mesh. The boundary physics codes were developed to permit design of divertor structures aimed at control of exhaust heat loads of tokamaks $[39,40]$. Simple scaling of results from existing devices indicated the heat loads were beyond available technology to remove the heat. It was therefore proposed to develop an improved operating mode in which radiation in the divertor region was enhanced to permit absorption of the exhaust heat over a larger surface area than that wetted by the SOL plasma $[41,42]$. The enhanced radiation was experimentally observed to lead to detached plasmas in which the plasma near the divertor floor was cooled sufficiently to permit three body recombination, thus reducing the ion flux to the plate, and the concomitant heat load. Successful modeling of the detachment process required accurate simulation of the neutral recycling process near the divertor plates, hence it was not sufficient to restrict the boundary codes to simple orthogonal meshes. Rather, one had to model geometries in which the divertor plates were inclined at small angles to the flux surfaces in the SOL. Furthermore, since detachment required not only power removal from the plasma, but also momentum removal, it was necessary to upgrade the UEDGE neutral model to include momentum exchange between ions and neutrals [33]. Early simulations of the detachment of plasmas in the DIII-D device demonstrated the importance of this momentum removal process as shown in Fig. 2 [43]. This figure shows that three-body recombination of exhaust ions only becomes significant when parallel flow of the plasma slows sufficiently that the recombination rate can compete with the ion flow to the plate. Thus the plasma velocity slows from Mach 1 near the ionization front several centimeters off the plate, to order 
Mach 0.1 near the plate, where the electron temperature is near $1 \mathrm{eV}$, and recombination processes dominate.

To successfully simulate the detachment process, one must include not only momentum removal processes, but power removal via radiation. Although in principle it is possible to detach the SOL plasma with only fuel ions and neutrals, it requires very high neutral densities in the divertor, and hence leads to large fueling of the plasma on closed surfaces via recycling. All existing devices have significant impurity radiation in the boundary region, so successful simulation of existing devices also requires modeling of impurity radiation. Early simulations used a simple impurity model in which the impurity density was assumed to be a specified fraction of the fuel ion density. The impurity species was specified, typically carbon for devices with carbon plasma facing walls, and the average charge state was determined from the local electron density and temperature. This fixed fraction model was sufficient to determine detachment boundaries in density and heating power for the DIII-D device [44]. These simulations were also compared with experimental measurements of the boundary plasma in detachment experiments, and it was shown that the UEDGE model was in reasonable agreement with experiment. Later, the impurity model was upgraded to permit simulation of all six ionization states of the intrinsic carbon impurity (or any other impurity which might be used in experiment) using a force balance model for the parallel transport of the impurity ions [45]. Further improvements in determination of the detachment boundaries of DIII-D were then made using these impurity models [46].

In addition to exploring the physics of detachment in existing experiments, the UEDGE code was used to assist design of advanced divertor configurations for the DIII-D experiment. Initially, the DIII-D divertor was a simple flat plate with carbon tiles designed to permit dissipation of the exhaust heat without damage. It was desirable to enhance the divertor design to permit control of the recycling neutrals, and thus better 
control of the neutral density fueling the core plasma. The UEDGE code was used to assist the design of this "Advanced Divertor Project" [47,48]. Code simulations indicated the neutral leakage from the divertor could be controlled with a baffle around the outer strike point. This baffling was expected to enhance the neutral density in the divertor region, and thus permit control of the confined plasma density by enabling neutral pumping with a cryogenic surface in a pumping baffle near the outer strike point. These density control expectations were born out with experiment after installation of the advanced divertor [49]. More recently, a baffle structure was designed, with guidance from UEDGE modeling, and installed in the upper divertor of DIII-D to permit pumping of high triangularity plasmas [50,51].

The modeling results described previously have not included the effect of classical plasma drifts arising from gradients in the magnetic field, and electric fields in the boundary region. The capability of including these effects in the model has recently been implemented [27,52]. It has been found that these effects are important, particularly in accurately determining the in/out asymmetries of the boundary plasma. It has even been speculated that these drift effects may play a role in determining the power threshold required to achieve plasma operation in the high-confinement mode (H-mode) [53]. Inclusion of these effects reproduce some of the measured flow patterns in existing devices, but not all $[54,55]$. Implementation of these drift effects for all operating modes of DIII-D is ongoing. Inclusion of the drift effects is crucial when modeling double null configurations $[56,57]$. The use of the double null configuration has been proposed as a means of increasing the wetted surface area over which power is exhausted in advanced tokamak modes [58]. However, it has been found experimentally that the up/down asymmetry of the power and particle exhaust is very sensitive to the up/down magnetic balance. UEDGE modeling, which is consistent with the experimental results, indicate 
that the in/out and up/down particle and power asymmetries are determined entirely by the effects of classical plasma drifts [57]. 


\section{INTERPRETIVE MODEL (OEDGE)}

The OEDGE code ("ㅁnion-Skin Modeling + EIRENE + ㅁIVIMP for edge analysis”) has been developed for interpretive analysis of the edge region with the aim of identifying, characterizing and quantifying the controlling physics processes. In this approach as much empirical data as possible is used as input. Monte Carlo codes are used to make most of the comparisons with experimental data. EIRENE is a neutral (hydrogen, etc.) Monte Carlo code [59]. DIVIMP is an impurity neutral and ion Monte Carlo sputtering and transport code [60]. The Monte Carlo codes require a "plasma background" into which to launch particles and this is provided by so-called onion-skin modeling, OSM see Chap. 12 in Ref. [1], which solves the 1-D plasma (fluid) conservation equations along the magnetic field $\mathbf{B}$, using across- $\mathbf{B}$ boundary conditions from experiment to produce a 2-D solution for the edge plasma. OSM analysis is not very sensitive to cross-field transport assumptions, e.g. as to whether it is diffusive or convective; also, transport coefficients such as the values of $\mathrm{D}_{\perp}$ and $\chi_{\perp}$ are not required as input in OSM.

The boundary conditions are usually taken to be at the target end (i.e. the "downstream" end) of each SOL flux tube, in the form of $T_{\mathrm{e}}$ and $n_{\mathrm{e}}$ at locations distributed across the targets. This information is also usually taken from experimental measurements, e.g. from target Langmuir probes or divertor Thomson scattering. Thus the solution-constraining information is input (a) from experiment and (b) across the SOL, radially — thereby constraining each separate flux tube in the SOL.

OSM analysis can be carried out at various levels of sophistication. At the most basic level, simple, analytic 1D SOL models are used. At a more sophisticated level, the full set of conservations equations [Eq. (1)], are used, in the 1D form, with the neutral hydrogen- 
related and impurity-related terms being provided iteratively as output from the Monte Carlo codes, in this case, the effect of cross-field transport appears as sources/sinks (deduced from overall balances for each flux tube) in the 1D conservation equations. For illustration, consider the particularly simple situation where each flux tube in the SOL can be assumed to be in the sheath-limited regime, with temperature constant along each flux tube, but varying radially as a result of the radial variation of the boundary values at the target. For each flux tube, simple analytic expressions then give the values of $T_{\mathrm{e}}, T_{\mathrm{i}}$, $n, v$ and $E$ at each location $s_{\|}$along the tube, measuring from the target along $\boldsymbol{B}$. Since $T_{\mathrm{e}}, T_{\mathrm{i}}$ and $n$ generally vary across the targets, the OSM Method thus generates a 2-D solution of the SOL. It may be seen that the information needed to give the radial variations at each location along the SOL entered the solution directly from the target boundary conditions. This information does not enter via specified cross-field transport coefficients $-D_{\perp}, \chi_{\perp}$, etc. - which are not imposed in the OSM approach. These coefficients can be extracted, in effect from the target boundary conditions, using the OSM analysis. For some applications one may need to have more freedom, namely to directly prescribe spatial variations of $n\left(s_{\|}\right), T_{\mathrm{e}, \mathrm{i}}\left(s_{\|}\right), v\left(s_{\|}\right), E\left(s_{\|}\right)$, etc. in which some or all of these quantities are arbitrarily prescribed without regard to whether they satisfy the conservation equations. Such flexibility can be useful when trying to identify what plasma background is implied to exist by measured hydrogenic and impurity spectroscopic signals and to thus identify the controlling physical processes, i.e. the terms needed in the conservation equations. This can be useful for complex regimes, such as divertor detachment [61], or the private flux zone [62], where it may not be clear at present how to model (using conservation equations) the features of the problem in adequate detail. 


\section{CODE VALIDATION AGAINST DIII-D EXPERIMENTS}

Development of the UEDGE boundary plasma simulation code has been driven in large part by the ability to validate the model against a very thorough diagnostic set on the DIII-D tokamak. These validation efforts were begun early, when the code was relatively crude, and the diagnostic set was limited, and has continued as both the code and diagnostics have improved. One of the diagnostics which have proven invaluable is the Thomson scattering measurements of electron density and temperature. The measurement which provides the profile on $n_{\mathrm{e}}$ and $T_{\mathrm{e}}$ in the confined region of the plasma extends into the SOL, providing determination of the density and temperature profiles near the separatrix with a spatial resolution of around $1.5 \mathrm{~cm}$ [63]. This important capability was augmented by the addition of the only working Thomson scattering measurements of the plasma profile in the divertor region [64-66]. The existence of data from both regions of the boundary plasma has proven valuable in assessing the models, as shown in Fig. 3 from [2]. The existence of the divertor Thomson scattering measurements were particularly valuable in demonstrating that the electron temperature of a detached plasma was near $1 \mathrm{eV}$ rather than 5 to $10 \mathrm{eV}$ as determined from Langmuir probe measurements in the divertor plates [67]. The lower temperature had been predicted by early modeling of the boundary layers $[44,68]$.

The code validation effort on DIII-D can be characterized by considering recent results in which the simulations are compared with a wide variety of experimental data from the boundary region [69]. Simulation of a particular discharge begins with estimation of the anomalous cross-field transport coefficients. These coefficients are not known from first principles, although progress is being made in this area as well [23]. One way to treat these unknown transport coefficients is to assume a simple model for thermal and particle transport which is diffusive, and characterized by a spatially constant 
diffusivity for three processes: electron thermal transport, ion thermal transport, and particle transport. Parallel transport in the boundary region is assumed classical [17], with flux limits imposed to account for kinetic effects. The UEDGE code is then run with these radial transport rates, and boundary conditions which derive from known heating processes. The radial transport rates are varied to obtain consistency between the simulated and measured radial profiles far upstream from the divertor in the outer midplane region, as shown in Fig. 4. Note that the simulations are done for steady state conditions, while the measurements of a H-mode plasma vary during the ELM cycle. The simulated profile is fit to the profile measured just before the onset of an ELM. It is rather remarkable that the measured profiles can be simulated with such simple radial transport models, i.e. the transport is characterized by a single number for each of the three processes. The density profile is determined by assumptions of the neutral particle removal rate (wall pumping) as well as the assumption of a particle diffusivity, $D$. Because of this, the choice of $D$ for the simulation is guided by the experimental result that the particle diffusivity is approximately one fourth of the thermal diffusivity $(D=\chi / 4)[70]$. Unfortunately, the experimentally determined plasma profiles of density and temperature upstream from the divertor region do not uniquely determine radial transport. The measured profiles can be fit equally well with a model which assumes the radial transport has a convective component which increases strongly as the chamber wall is approached, rather than being purely diffusive [71,72].

The ability to reproduce the radial profiles of the upstream plasma is not a real test of the efficacy of the physics models in comprehensive boundary region codes such as UEDGE. Rather, they are a means to estimate some parameters which are uncertain in experiments, such as radial transport, and particle removal rates. More relevant tests of the code arise when the results obtained in the simulations are compared with all existing experimental measurements in the boundary region. These include a large variety of 
diagnostics on the DIII-D device, including profiles of various line emissions, profiles of radiated power, divertor plasma characteristics measured with Thomson scattering and probes, 2D images of line radiation, etc. Thus the efficacy of the comprehensive model is assessed by comparing the simulations which are consistent with the upstream plasma profiles with other data. The comparison between experiment and simulation for some of the DIII-D diagnostic data is shown in Fig. 5 (from Ref. [69]) for the simulation shown in Fig. 4. The diagnostics measured in the divertor region are reasonably matched by the simulation. (The discrepancy with the density measured by the divertor Thomson scattering was found to arise from an inaccurate calibration of the data for absolute density.) The simulated $\mathrm{D}_{\alpha}$ emission in the private region is frequently significantly lower than measured, as seen by the emission at 1.3 to $1.4 \mathrm{~m}$ in Fig. 5. This discrepancy is generally largest in simulations of H-mode plasmas and is not understood. Simulations of L-mode plasmas, including the effect of cross field drifts, are much more consistent with measured $\mathrm{D}_{\alpha}$ emission profiles. The simulation was compared with additional diagnostics in the original paper. The agreement between simulation and experiment suggests the model contains much of the physics relevant to understanding boundary region plasmas.

The simulations which are compared with the H-mode plasma results in Ref. [69] do not include the effect of drifts. Understanding plasma flows is believed to be important in providing control of both neutral fuel particles, and impurity ions. Control of impurities is necessary for successful design of divertors operating with detached plasmas. The discharge described in this section included early measurements of these flows, using spectroscopic techniques described in Ref. [73]. This diagnostic measures the line integral of line emission seen by viewing the divertor tangentially, and infers the parallel velocity of impurity ionization states at two radial positions by determining the Doppler shift and Zeeman split of the emission. The UEDGE simulations compared favorably with these early measurements, as shown in Fig. 6, suggesting the UEDGE model is 
relevant to the plasma flows, even without the effect of drifts. The simulation is compared with data obtained from one of the tangentially viewing flow diagnostics. The view of this channel, projected to a poloidal plane, is shown by the solid line in Fig. 6(c). However, the spatial resolution of the experimental measurement does not provide a definitive test of the physics models, so work continues to better understand these plasma flows. Recent simulations indicate the drifts have a strong effect on the divertor plasma, particularly on the high field side which is in general more poorly diagnosed [57]. 


\section{INTERPRETATIVE ANALYSIS OF DIII-D EDGE PLASMAS USING OEDGE}

In recent years a number of unanticipated major effects have shown up in tokamak edge research, including high tritium retention, strong plasma-wall contact, narrow target power profiles and fast parallel flows. Undoubtedly many parts of our earlier picture remain correct. The task is to verify which parts and to continue to identify the controlling physics in the edge. This has motivated the simplest-as-possible plasma (SAPP) studies recently undertaken on DIII-D. The approach:

1. Start with simplest possible conditions, e.g. no ELMs, no detachment.

2. Operate as comprehensive a set of edge diagnostics as possible.

3. Run many repeat shots.

4. Do not put aside any diagnostic unless it is known to be misfunctioning.

5. Bring all of the measurements into mutual, detailed comparison with an interpretive edge code, such as OEDGE.

6. If most of the data are matched by the code, then we may conclude that probably the controlling physics has been correctly identified and quantitatively characterized.

7. Any outstanding discrepancies - including apparently incomprehensible measurements - are to be flagged for further investigation as potentially containing important information.

8. Proceed to more complex regimes.

A set of 10 identical, low density, $\bar{n}_{\mathrm{e}} \sim 2 \times 10^{19} \mathrm{~m}^{-3}$, attached, L-mode, lower singlenull divertor shots on DIII-D with $1 \mathrm{MW}$ total input power were taken as the initial SAPP candidate [3]. The first focus was on the plasma at the outer target, where the edge 
diagnostics are particularly comprehensive on DIII-D. The location of the outer strike point was swept radially during the flattop parts of the shots. The value of $T_{\mathrm{e}}$ measured by the divertor Thomson scattering system (DTS), dropped sharply at the transition from common to private flux side of the separatrix to the SOL side - generally to within $<1 \mathrm{~cm}$. This constitutes an important test of the validity of the EFIT reconstruction of the magnetic equilibrium. The whole analysis procedure is dependant on the reliability of the computational grid, Fig. 7, generated from the EFIT equilibrium, since it is required for making mutual comparisons of the various data at different poloidal locations.

Figure 8 shows the profiles of $T_{\mathrm{e}}$ and $I_{\text {sat }}^{+}$measured across the outer target using the built-in Langmuir probes, as a function of the normalized magnetic flux coordinate $\Psi_{\mathrm{n}}$ [ $\Psi_{\mathrm{n}}=1$ at the separatrix; $\Psi_{\mathrm{n}}>1(<1)$ on the common (private) flux side], combining data from 6 probes for each shot and repeated for 10 shots. The divertor plasma was found to be constant while being swept, as evidenced by the different probes, which sample the $T_{\mathrm{e}}$ and $I_{\text {sat }}^{+}$profiles at different times but give indistinguishable profiles. The Thomson $T_{\mathrm{e}}$ data showed a peak target value of about $20 \mathrm{eV}$, lower than the probe value of about $35 \mathrm{eV}$. The quantity and quality of both the Thomson scattering spectra and probe data were exceptional - perhaps even uniquely so in tokamak studies - and therefore this discrepancy, which exceeds the scatter and known errors, and is as yet unexplained, has to be flagged as one of potential importance, evidently pointing to a significant deficiency in our basic understanding of sheath physics in the tokamak environment. SAPP studies at higher density, $\bar{n}_{\mathrm{e}} \sim 4 \times 10^{19} \mathrm{~m}^{-3}$, strongly reinforced this conclusion: peak $T_{\mathrm{e}}$ values measured by the Langmuir probes were $\sim 15 \mathrm{eV}$, while DTS measured $\sim 0.8 \mathrm{eV}$, the latter being quite well supported by the hydrogenic emissivity at the target, which is highly sensitive to $T_{\mathrm{e}}$ for very cold plasmas. The Langmuir probe current-voltage characteristics appeared to be completely normal, "classical", and there is no obvious justification for ignoring these data. At the same time, Thomson scattering 
spectra indicated a "well thermalized" Gaussian electron energy distribution. The present conclusion is that the probe " $T_{\mathrm{e}}$ " measurements contain real - and probably quite important information - but we do not know how to interpret these data at this time. It is likely that the high probe " $T_{\mathrm{e}}$ " values contain information about the sheath drop at the target, thus the ion impact energy, sputtering yield and power deposition - all matters of major practical importance. Fortunately, the probe $I_{\text {sat }}$ values appear to be reliable, including high density regimes and detachment: the excellent agreement between the code and the hydrogenic emissivities found in the high density SAPP studies were as much a confirmation of the probe $I_{\text {sat }}$ values as the DTS $T_{\mathrm{e}}$ values. This is a most valuable confirmation that our ideas about the flux of positively charged particles reaching negatively charged solid surfaces are valid for the tokamak environment. This plasma quantity is even more fundamental to our understanding of plasma-surface interactions than the sheath potential drop.

The DTS $T_{\mathrm{e}}$ and the probe $I_{\text {sat }}^{+}$were used as OSM boundary conditions. Figure 7 shows the poloidal flux surfaces, i.e. the "onion-rings". Figure 9 shows comparisons of OEDGE output with the DTS data for eight outer SOL rings identified by their $\Psi_{\mathrm{n}}-$ number. $s_{\|}$is the distance along $\boldsymbol{B}$ from the target. Figure 10 shows code comparisons with the absolutely calibrated filterscope (FS), and multi-chord divertor spectrometer (MDS) outer target profiles of $D_{\alpha}, D_{\beta}, D_{\gamma}, \mathrm{CI}, \mathrm{CII}$ and CIII. Figure 11 shows code comparisons with the "upstream" radial profiles measured by the main Thomson system and the reciprocating probe; although the DTS $\mathrm{T}_{\mathrm{e}}$ values were used here as boundary conditions for the OSM solution, the calculated upstream quantities are not greatly altered if the target probe $T_{e}$ values are used, since when calculating in the 'upstream direction', solutions are rather insensitive to almost all input assumptions - a useful feature of the OSM approach [1]). (Figures 9 through 11 are for a $\bar{n}_{\mathrm{e}} \sim 2 \times 10^{19} \mathrm{~m}^{-3}$ case). The horizontal coordinate in Fig. 10 corresponds to the FS or DTS line-of-sight to 
the point on the target where the $\Psi_{\mathrm{n}}$-flux line strikes the target. The close agreement between code and experiment in Figs. 9 through 11, evidently indicates that most of the controlling physics has been correctly identified and incorporated in the model - at least for this simplest plasma condition. As noted, however, even here an important piece of missing physics has been identified, namely, a basic deficiency in our understanding of sheath physics in the tokamak environment, as manifest by a major discrepancy in two fundamental diagnostics of the plasma electrons, Langmuir probes and Thomson scattering.

There was no detectible carbon-deuteron (CD) band radiation at the outer target, evidently indicating the absence of chemical sputtering; $C_{2}$ (carbon-carbon) band radiation was also very weak. The assumption made in the DIVIMP code, therefore, was that physical sputtering, including self-sputtering, was the sole release mechanism for carbon at the outer target. The normal incidence sputtering yields were doubled to allow for estimated average incidence angles of $\sim 45 \mathrm{deg}$, a common modeling assumption. The effective temperatures from the line widths measured by MDS viewing the outer target are compared with the code output in Table I. The code value for the C-atom (CI) temperature, $1.25 \mathrm{eV}$, is the result for a Thompson speed distribution for physically sputtered neutrals, truncated for an assumed $D^{+}$impact energy of $100 \mathrm{eV}\left(=5 \mathrm{kT}_{\mathrm{e}, \text { target }}\right)$, a cosine angular distribution, and allowing for the viewing angle of the MDS spectrometer. The truncated Thompson energy distribution is given by $f(E) \propto\left[E /\left(E+E_{\mathrm{B}}\right)^{3}\right]\left\{1-\left[\left(E+E_{\mathrm{B}}\right) / \gamma(1-\gamma) E_{\text {impact }}\right]^{1 / \angle}\right\}$, where $E_{\mathrm{B}}=7.4 \mathrm{eV}$ is the $C$ binding energy and $\gamma=4 m_{\mathrm{D}} m_{\mathrm{C}} /\left(m_{\mathrm{D}}+m_{\mathrm{C}}\right)^{2}$. The good agreement between code and experiment, with regard to the CI, CII and CIII lines supports the hypothesis that chemical sputtering is not playing a significant role at the outer divertor target in DIII-D. There is, however, an inconsistency with regard to the Doppler shift of the CI line: the experimental shift indicates an average velocity of the $C$-atoms away from the target 
surface of $\sim 1.8 \mathrm{~km} / \mathrm{s}$, while the truncated Thomson distribution gives $\sim 6 \mathrm{~km} / \mathrm{s}$. The WBC impurity code has been used to calculate the velocity distribution resulting from the breakup of hydrocarbon molecules produced by chemical sputtering for the same plasma conditions [74]; the calculated Doppler shift is closer to experiment, $\sim 1.3 \mathrm{~km} / \mathrm{s}$, however the effective temperature is too high, $\sim 3 \mathrm{eV}$. Further SAPP studies are proceeding to resolve this remaining discrepancy: (a) studies using He SAPP plasmas where chemical sputtering cannot occur and which will therefore test the validity of the truncated Thomson distribution for physical sputtering for the operating conditions at the outer divertor target in DIII-D, (b) studies using a porous plug methane injector (DiMES) will provide experimental measurements of the Doppler shift and effective temperature of $C$ atoms resulting from breakup of hydrocarbon molecules. It is not known why the outer divertor target should be resistant to chemical sputtering, but the very prolonged exposure to intense plasma conditioning and/or multiple boronizations, which have been experienced by the outer target tiles in DIII-D, may be involved. The practical implications of such resistance strongly motivates the resolution of this matter. 


\section{SUMMARY and future directions}

The development of comprehensive models of the plasma region which extends from the top of an H-mode pedestal, on the closed field lines, across the separatrix to the plasma facing material surfaces has been described. These models have been powerful tools which enable development of a complete picture of the physics of this important region of a tokamak device which is consistent with the diagnostic information available. The codes have also been useful in guiding design of effective divertor structures on DIII-D which have successfully controlled recycling neutrals, and impurities sputtered from the carbon tiles facing the hot plasma. These divertors have permitted effective control of the confined plasma density for both low triangularity (ITER-like) and high triangularity Advanced Tokamak (AT) plasmas. The plasma models have been validated thoroughly against the extensive set of boundary layer diagnostics in DIII-D. The codes used to model the DIII-D boundary plasma use fluid plasma equations with flux limits to avoid excessive fluxes arising from kinetic effects. These fluid equations do not pertain at the top of the edge pedestal in the best confinement discharges in DIII-D indicating a need for kinetic codes for analysis of the boundary of AT operation. The models are not fully predictive because of uncertainties in the character of cross field transport, and its scaling. Experimental measurements can be reproduced using different models for this turbulence driven transport, diffusive or convective. The ability to determine the turbulence driven transport from first principles is being developed, and incorporated into the comprehensive models.

Interpretive analysis of DIII-D divertor measurements made in simple-as-possible plasmas has identified two unresolved issues of potential significance. Langmuir probe $\mathrm{T}_{\mathrm{e}}$ values can differ from Thomson scattering and spectroscopically-inferred values by very large factors, $>10$. The probe IV characteristics appear to be normal and thus this 
discrepancy may contain useful information if we can learn how to interpret it. There is substantial - although not totally consistent - evidence that the graphite tiles at the outer divertor target in DIII-D have been largely "passivated" to chemical sputtering an effect of great practical importance if it can be conclusively established. 


\section{Acknowledgments}

This is a report of work supported by the U.S. Department of Energy under W-7405ENG-48 with UC, LLNL and DE-FC02-01ER54698.

This paper summarizes the work done by the DIII-D program in this area over the past twenty years or more and includes the contributions of many members of the DIII-D Team listed in the Appendix of this volume of Fusion Science and Technology. 


\section{References}

[1] P.C. Stangeby, The Plasma Boundary of Magnetic Fusion Boundaries, Plasma Physics, Ed. P. Stott and H. Wilhelmsson. 2000 (Bristol: Institute of Physics Publishing).

[2] R.D. Stambaugh, G. Janeschitz, S. Cohen, D. Hill, et al., Nucl. Fusion 39, 2391 (1999).

[3] P.C. Stangeby, J.A. Boedo, B.D. Bray, J.D. Elder, et al., J. Nucl. Mater. 313-316, 883 (2003).

[4] R. Maingi, Nucl. Fusion 34, 283 (1994).

[5] L.W. Owen, R. Maingi, D.K. Lee, P.K. Mioduszewki, et al., J. Nucl. Mater. 220222, 315 (1995).

[6] J.T. Hogan, R. Maingi, P.K. Mioduszewski, L.W. Owen, et al., J. Nucl. Mater. 241-243, 612 (1997).

[7] D.R. Baker, R. Maingi, L.W. Owen, G.D. Porter, et al., J. Nucl. Mater. 241243, 602 (1997).

[8] B.A. Carreras, L.W. Owen, R. Maingi, P.K. Mioduszewski, et al., Phys. Plasmas 5, 2623 (1998).

[9] L.W. Owen, B.A. Carreras, and R.M. Maingi, Plasma Phys. Control. Fusion, 40, 717 (1998).

[10] L.W. Owen, B.A. Carreras, R. Maingi, P.K. Mioduszewski, et al., J. Nucl. Mater. 266-269, 890 (1999).

[11] L.W. Owen, R.J. Colchin, R. Maingi, and M.E. Fenstermacher, J. Nucl. Mat.er, 290-293, 464 (2001).

[12] R. Maingi, L.W. Owen, R.J. Colchin, M.E. Fenstermacher, et al., "Modeling of 2D Neutral Density Measurements in DIII-D," Proc. 26 ${ }^{\text {th }}$ Euro. Physical Society 
Conference on Controlled Fusion and Plasma Physics, Maastricht, Netherlands, 1999 (European Physical Society, Petit-Lancy).

[13] L.L. Lao, H.E. St John, R.D. Stambaugh, A.G. Kellman, et al., Nucl. Fusion 25, $1611(1985)$.

[14] T.D. Rognlien, J. Milovich, M. Rensink, and G.D. Porter, “J. Nucl. Mater. 196-198, 347 (1992).

[15] T.D. Rognlien, P.N. Brown, R.B. Campbell, T.B. Kaiser, et al., "Contr. Plasma Phys. 34, 362 (1994).

[16] P.N. Brown and A.C. Hindmarsh, SIAM J. Numer. Anal. 23, 610 (1986).

[17] S.I. Braginskii, Transport Processes in a Plasma, in Reviews of Plasma Physics, M.A. Leontovich, Ed. (Consultants Bureau: New York, New York, 1965) p. 205.

[18] J. Neuhauser, W. Schneider, R. Wunderlich, K. Lackner, et al., Nucl. Fusion 24, 39 (1984).

[19] R.H. Cohen and T.D. Rognlien, Contrib. Plasma Phys. 34, 198 (1994).

[20] G. Radford, "Classical Parallel Transport in a Multi-Species Plasma from a 21 Moment Approximation,” Joint European Torus Report JET-R(93)05 (1993).

[21] A. Bergmann and Y. Igitkhanov, Contrib. Plasma Phys. 36, 192 (1996).

[22] X. Xu and R.H. Cohen, “ Plasma Phys. 36, 202 (1996).

[23] X. Xu, R.H. Cohen, W.M. Nevins, G.D. Porter, et al., Nucl. Fusion 42, 21 (2002).

[24] X. Xu, R.H. Cohen, G.D. Porter, T.D. Rognlien, et al., "Nucl. Fusion 40, 731 (2000).

[25] T.D. Rognlien, M.V. Umansky, X.Q. Xu, and R.H. Cohen, "Self-Consistent Simulation of Turbulence and Transport in Tokamak Edge Plasmas," to be published in Contrib. Plasma Phys. (2004).

[26] X.Q. Xu, W.M. Nevins, R.H. Cohen, T.D. Rognlien, et al., "Correction of Density Pedestal Width and Neutral Penetration Length," to be published in Contrib. Plasma Phys. (2004). 
[27] T.D. Rognlien, G.D. Porter, and D.D. Ryutov, “J. Nucl. Mater. 266-269, 654 (1999).

[28] R.K. Janev, D.E. Post, W.D. Langer, K. Evans, et al., “J. Nucl. Mater. 121, 10 (1984).

[29] J. Weisheit, J. Physics B (Atomic and Molecular Physics) 8, 2556 (1975).

[30] R.V. Jensen, D.E. Post, W.H. Grasberger, C.B. Tarter, et al., Nucl. Fusion 17, 1187 (1977).

[31] H.P. Summers and M.v. Hellermann, Atomic and Plasma Material Interaction Processes in Controlled Thermonuclear Fusion, R. Janev and H. Drawin, Eds (Elsevier: Amsterdam, 1993) p. 87.

[32] M.E. Rensink, L. Lodestro, G.D. Porter, T.D. Rognlien, et al., Contrib. to Plasma Phys. 38, 325 (1998).

[33] F. Wising, D.A. Knoll, S.I. Krasheninnikov, T.D. Rognlien, et al., Contrib. Plasma Phys. 35, 136 (1996).

[34] D. Reiter, C. May, D. Coster, and R. Schneider, J. Nucl. Mater. 220-222, 987 (1995).

[35] D.A. Knoll, S.I. Krasheninnikov, P.R. McHugh, and D.J. Sigmar, Phys. Plasmas 3, 3358 (1996).

[36] R.K. Janev and H.W. Drawin, Eds, Atomic and Plasma-Material Interaction Processes in Controlled Thermonuclear Fusion (Elsevier: Amsterdam, 1993) p. 484.

[37] W.O. Hofer and J. Roth, Physical Processes of the Interaction of Fusion Plasmas with Solids, W.O. Hofer and J. Roth, Eds (Academic Press) 1966.

[38] B. Braams, NET, EUR-FU/XII-80/87/68 (1987).

[39] G. Janeschitz, A. Antipenkov, S. Chiocchio, J. Dietz, et al., "The Physics Basis and Design of the ITER Divertor and of the Pumping and Fueling System," in 
Proc. of $15^{\text {th }}$ International Conference on Plasma Physics and Controlled Nuclear Fusion Research, Seville, Spain, 1994 (IAEA, Vienna).

[40] G. Janeschitz, K. Borrass, G. Federici, Y. Igitkhanov, et al., "J. Nucl. Mater. 220-222, 73 (1995).

[41] P.-H. Rebut, D. Boucher, D.J. Gambier, B.E. Keen, et al., “The ITER Challenge,” in Proc. of $17^{\text {th }}$ Symposium on Fusion Technology, Rome, Italy, 1992.

[42] M. Watkins and P.-H. Rebut, "Energy Exhaust Through Neutrals in a Tokamak Divertor," in Proceedings of the $19^{\text {th }}$ European Conference on Controlled Fusion and Plasma Physics, Innsbruck, Austria, 1992.

[43] G.D. Porter, S. Allen, M.E. Fenstermacher, D. Hill, et al., "Divertor Characterization Experiments and Modeling in DIII-D," in Proceedings of $23^{\text {rd }}$ European Conference on Controlled Fusion and Plasma Physics, Kiev, Ukraine, 1996 (European Physical Society, Petit-Lancy).

[44] G.D. Porter, S. Allen, M. Brown, M.E. Fenstermacher, et al., Phys. Plasmas 3, 1967 (1996).

[45] G.R. Smith, P.N. Brown, R.B. Campbell, D.A. Knoll, et al., "J. Nucl. Mater. 220-222, 1024 (1995).

[46] N.S. Wolf, G.D. Porter, D.N. Hill, and S.L. Allen, J. Nucl. Mater. 269, 739 (1999).

[47] S.L. Allen, M.E. Rensink, D. Hill, R. Wood, et al., J. Nucl. Mater. 196-198, 804 (1992).

[48] S.L. Allen, N.H. Brooks, R.B. Campbell, M.E. Fenstermacher, et al., J. Nucl. Mater. 220-222, 336 (1995).

[49] M.A. Mahdavi, S.L. Allen, D.R. Baker, B. Bastasz, et al., J. Nucl. Mater. 220-222, 13 (1995).

[50] S.L. Allen, J.A. Boedo, A.S. Bozek, N.H. Brooks, et al., J. Nucl. Mater. 290, 995 (2001). 
[51] S.L. Allen, M.E. Fenstermacher, C.M. Greenfield, A.W. Hyatt, et al., J. Nucl. Mater. 269, 168 (1999).

[52] T.D. Rognlien, D.D. Ryutov, N. Mattor, and G.D. Porter, Phys. Plasmas 6, 1851 (1999).

[53] M.J. Schaffer, B.D. Bray, J.A., Boedo, T.N. Carlstrom, et al., Phys. Plasmas 8, 2118 (2001).

[54] G.D. Porter, J.A. Boedo, T.N. Carlstrom, R.J. Groebner, et al., "Modeling the Effects of Drifts on the Edge, Scrape-Off Layer and Divertor Plasma in DIII-D," in Proceedings of $28^{\text {th }}$ European Conference on Controlled Fusion and Plasma Physics, Madeira, Portugal, 2001 (European Physical Society).

[55] G.D. Porter, T.D. Rognlien, M.E. Rensink. A. Loarte, et al., "J. Nucl. Mater. 313, 1085 (2003).

[56] M.E. Rensink, S.L. Allen, G.D. Porter, and T.D. Rognlien, Contrib. to Plasma Phys. 40, 302 (2000).

[57] M.E. Rensink, C.J. Lasnier, T.W. Petrie, G.D. Porter, et al., Contrib. Plasma Phys. 42, 181 (2002).

[58] D. Meade, "Mission and Design of the Fusion Ignition Research Experiment (FIRE)," in Proceedings of $18^{\text {th }}$ International Conference on Controlled Fusion, Sorrento, Italy, 2002 (IAEA).

[59] D. Reiter, "Neutral Gas Transport in Fusion Devices: Atomic and Surface Data Aspects," in Atomic and Plasma-Material Interaction Processes in Controlled Thermonuclear Fusion, R. Janev and H. Drawin, Eds. (Elsevier: Amsterdam, 1993) p. 243.

[60] P.C. Stangeby and J.D. Elder, Nucl. Fusion 35, 1391 (1995).

[61] S. Lisgo, P.C. Stangeby, J.D. Elder, B.D. Bray, et al., "Re-construction of Detached Divertor Plasma Conditions in DIII-D using only Spectroscopic and 
Probe Data," Proc. $16^{\text {th }}$ Int. Conf. on Plasma Surface Interactions with Controlled Fusion Devices, Portland, Maine, 2004, to be published in J Nucl Mater.

[62] S. Lisgo, et al,. "OEDGE Modeling of Neutral Pressures in the Alcator C-Mod Divertor," Proc. $16^{\text {th }}$ Int. Conf. Plasma Surface Interactions on Controlled Fusion Devices, Portland Maine, 2004; to be published in J Nucl Mater.

[63] T.N. Carlstrom, G.L. Campbell, J.C. DeBoo, R. Evanko, et al., Rev. Sci. Instrum. 63, 4901 (1992).

[64] S.L. Allen, D.N. Hill, T.N. Carlstrom, D.G. Nilson, et al., "J. Nucl. Mater. 241, 595 (1997).

[65] D.G. Nilson, T.N. Carlstrom, D.N. Hill, C.-L. Hsieh, et al., "Fusion Eng. and Design 34, 609 (1997).

[66] T.N. Carlstrom, J.H. Foote, D.G. Nilson, and B.W. Rice, Rev. Sci. Instrum. 66, 493 (1995).

[67] M.E. Fenstermacher, S.L. Allen, D.N. Hill, A.W. Leonard, et al., Contrib. Plasma Phys. 36, 127 (1996).

[68] M. Petravic, G. Bateman, and D. Post, "Contrib. Plasma Phys. 34, 381 (1994).

[69] G.D. Porter, R.C. Isler, J.A. Boedo, and T.D. Rognlien, Phys. Plasmas 7, 3663 (2000).

[70] G.D. Porter and DIII-D Team, Phys. Plasmas 5, 4311 (1998).

[71] A.Y. Pigarov, S.I. Krasheninnikov, T.D. Rognlien, M.J. Schaffer, et al., Phys. Plasmas 9, 1287 (2002).

[72] A.Y. Pigarov, S.I. Krasheninnikov, W.P. West, T.D. Rognlien, et al., J. Nucl. Mater. 313, 1076 (2003).

[73] R.C. Isler, N.H. Brooks, W.P. West, A.W. Leonard, et al., Phys. Plasmas 6, 541 (1999). 
[74] D.G. Whyte, J.N. Brooks, P.C. Stangeby, and N.H. Brooks, "A Detailed Study of Carbon Chemical Erosion in L-mode Plasmas in the DIII-D Divertor," in Juelich Carbon Workshop, Juelich, Germany, 2003, Physica Scripta. 
Table I. Effective temperatures (eV) from Doppler widths compared with OEDGE (DIVIMP) code values.

\begin{tabular}{lcc}
\hline \hline & Experiment & Code \\
\hline CI $(910 \mathrm{~nm})$ & $\sim 1$ & 1.25 \\
CII $(657.9 \mathrm{~nm})$ & $6-7$ & 6.9 \\
CIII $(465 \mathrm{~nm})$ & $10-15$ & 14.5 \\
\hline \hline
\end{tabular}




\section{List of Figure Captions}

Fig. 1. Magnetic configuration of a DIII-D double-null plasma. The flux surfaces are shown in the boundary region which is modeled as described in this section.

Fig. 2. Poloidal variation of plasma parameters near the separatrix on the inner leg, as simulated in UEDGE with a neutral model that includes parallel momentum exchange between the ions and neutrals. The parallel Mach number at the plate is set to be 1.0 as a boundary condition. The abscissa extends from the inner divertor plate to just below the X-point.

Fig. 3. Comparison of simulated (UEDGE) density and temperature profiles with experimental measurements near the outer midplane (left), and in the divertor region (right) for the DIII-D experiment. The midplane profiles are plotted versus distance from the separatrix at the midplane, while the divertor profiles are two dimensional plots vs $(\mathrm{R}, \mathrm{Z})$ at the divertor region.

Fig. 4. Comparison of simulated and experimental profiles of the electron density (a) and temperatures (b). The data is projected to the outer midplane, and is plotted as a function of the distance from the separatrix.

Fig. 5. Comparison of simulated and measured profiles in the divertor region for the same discharge shown in Fig. 4. The parameter $\delta Z$ is the distance above the floor at a major radius of $1.47 \mathrm{~m}$, the position of the DTS measurement. The lower right panel shows the radial variation of the line integrated $D_{\alpha}$ emission, $\varepsilon$, as seen by an array viewing the divertor floor from the top of the device. 
Fig. 6. Simulated total emission, $\varepsilon$, of the $6581 \AA$ doublet of CII along the upper and lower halve of one flow diagnostic view (a), the simulated and experimental parallel velocity, $u_{\|}$, of the CII ion (b), and the projection of the diagnostic view onto a poloidal plane shown by a solid line (c).

Fig. 7. Computational grid showing poloidal flux surfaces - the "onion-skin rings".

Fig. 8. Langmuir probe outer target profiles of $T_{\mathrm{e}}$ and the ion saturation current, $I_{\mathrm{s}}^{+}$.

Fig. 9. Profiles of $n_{\mathrm{e}}$ and $T_{\mathrm{e}}$ as functions of distance-along- $B\left(s_{\|}\right)$starting at the target for a number of computational "rings". Ring No. 15 (22) is the light (dark) shaded flux tube. Data (points). Code (lines). Combined divertor Thomson scattering data for 10 shots.

Fig. 10. Filterscope (FS) and multi-chord divertor spectrometer (MDS) profiles across the outer target. Data (points) Code (lines).

Fig. 11. Comparison of OEDGE code results for the "upstream" radial profiles of $n_{\mathrm{e}}$ and $T_{\mathrm{e}}$ measured by Thomson scattering and reciprocating probe. The abscissa $\Psi_{n}$ is the normalized poloidal flux. The curves are derived from the Onion Skin Model (OSM), reciprocating probe (RCP), and Thomson scattering data (Thomson). 


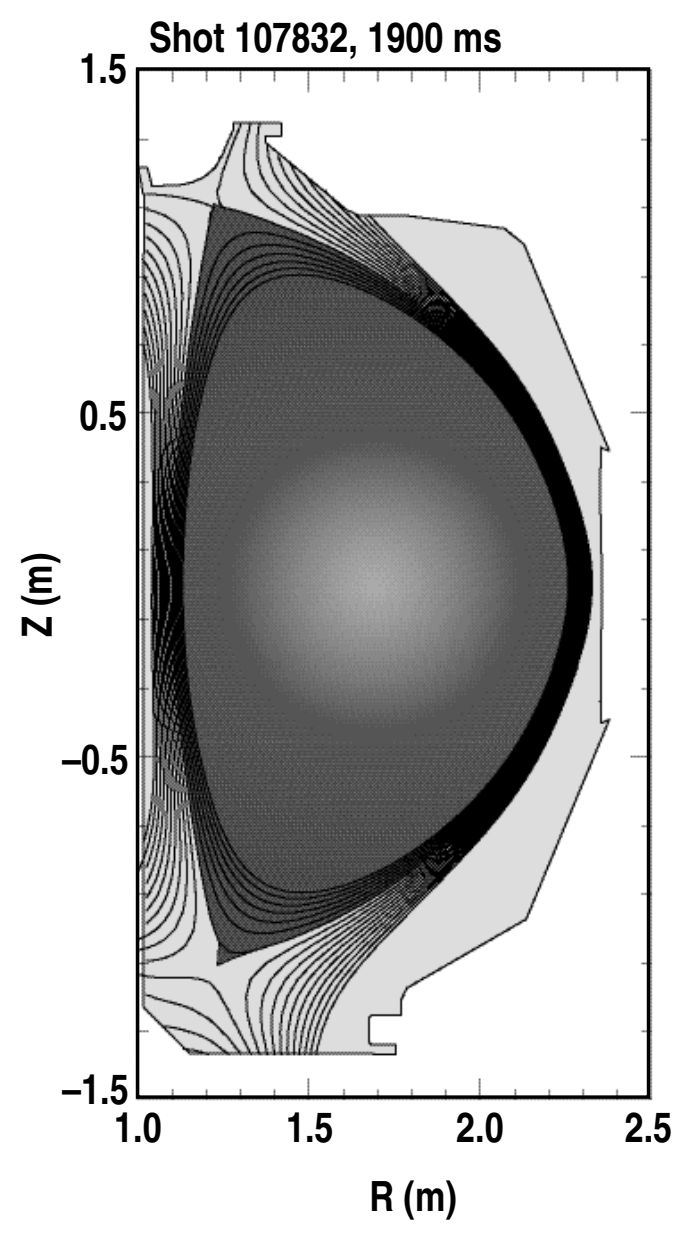

G.D. Porter Figure 1 

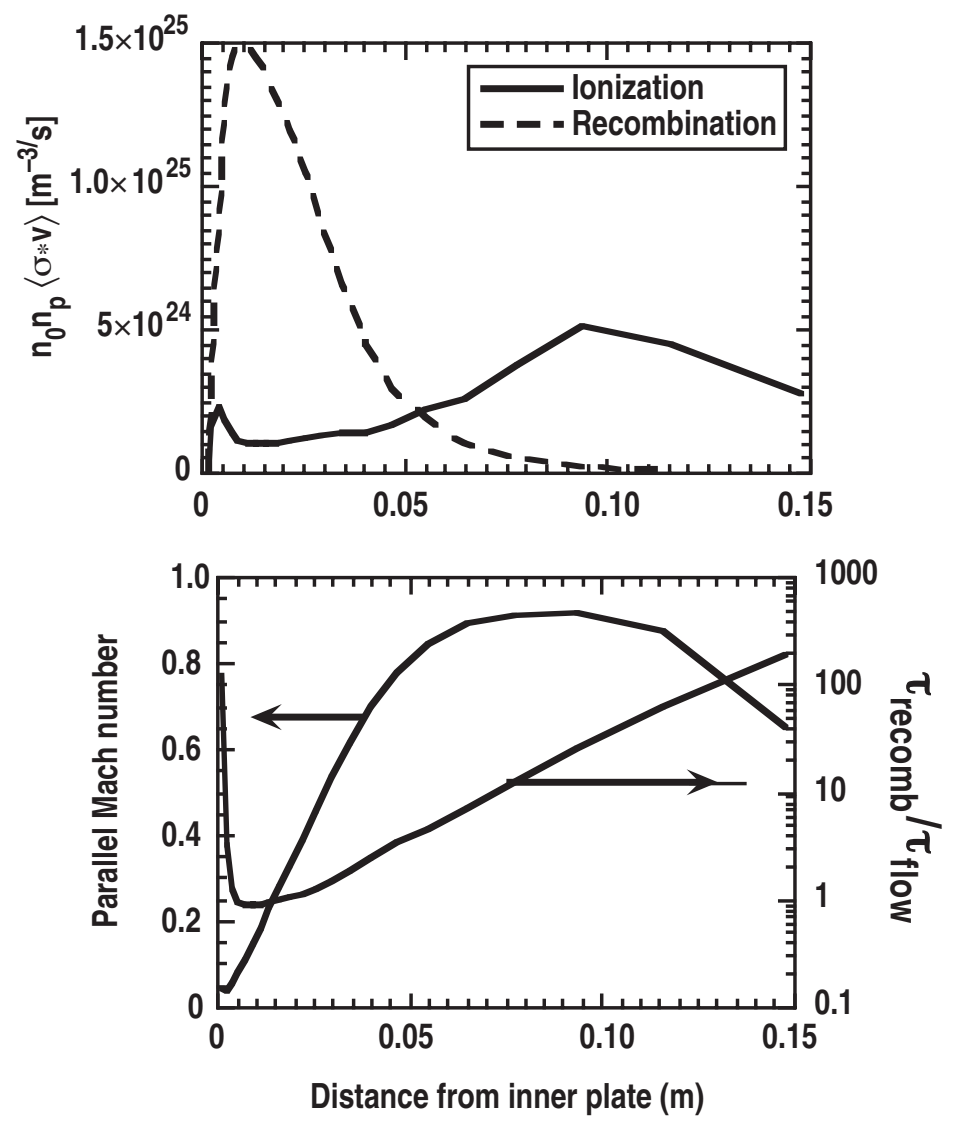

G.D. Porter Figure 2 

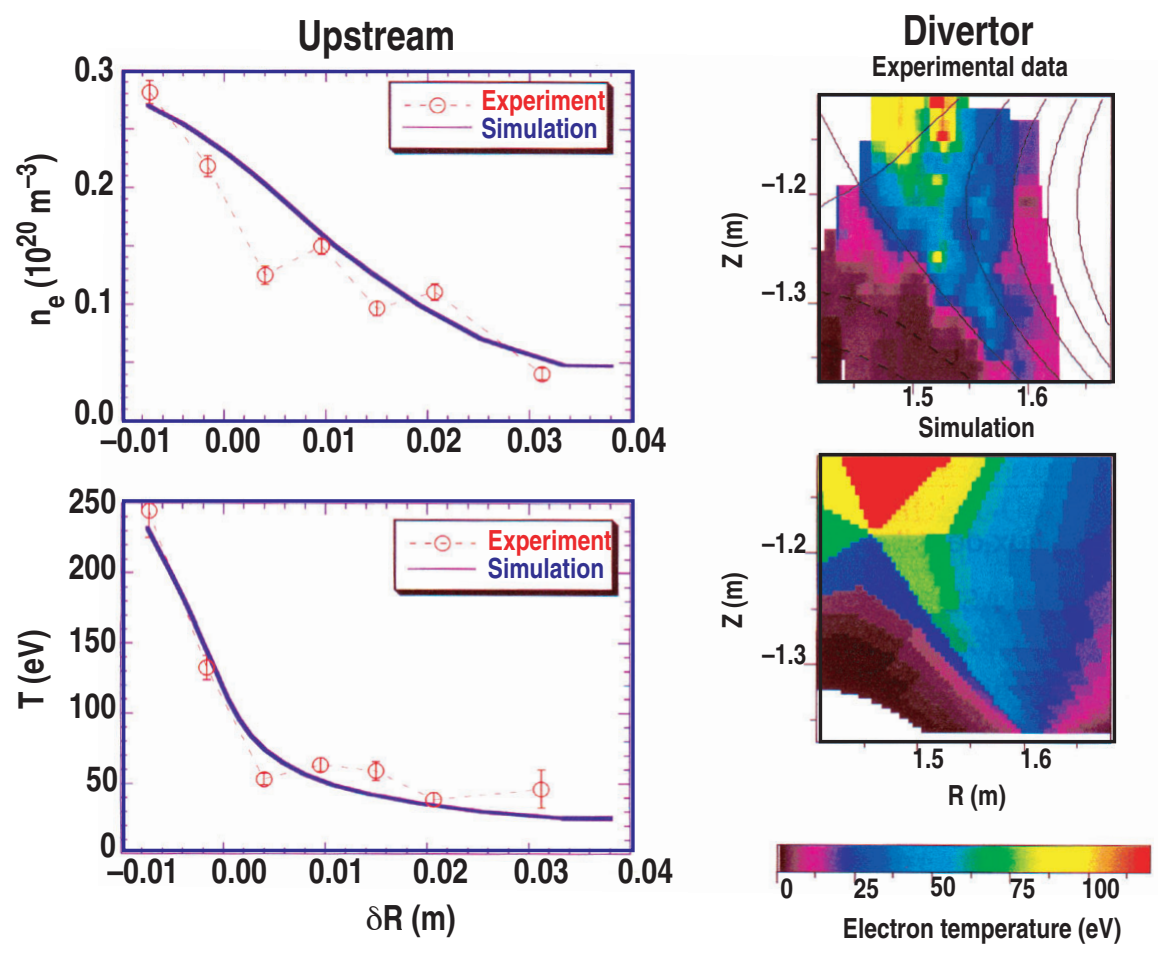

G.D. Porter Figure 3 

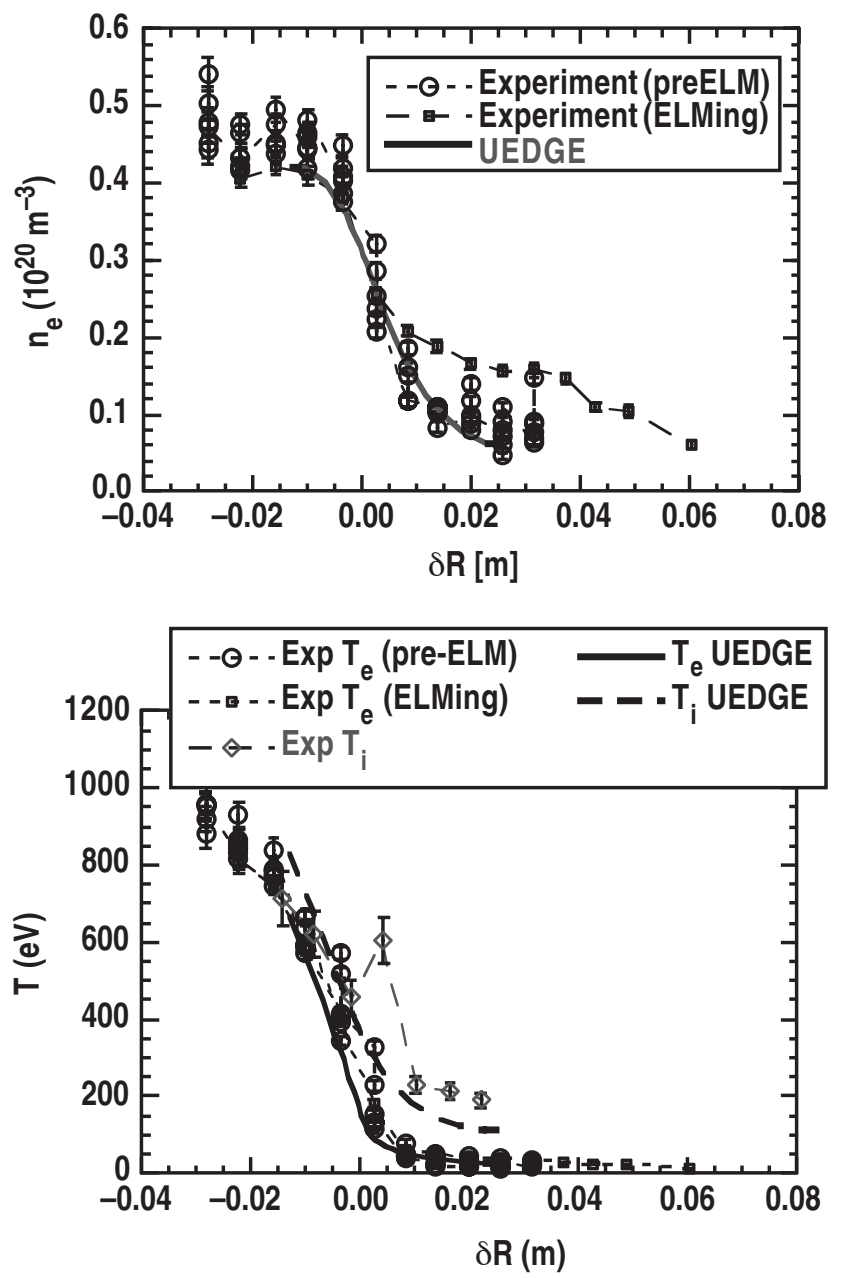

G.D. Porter Figure 4 

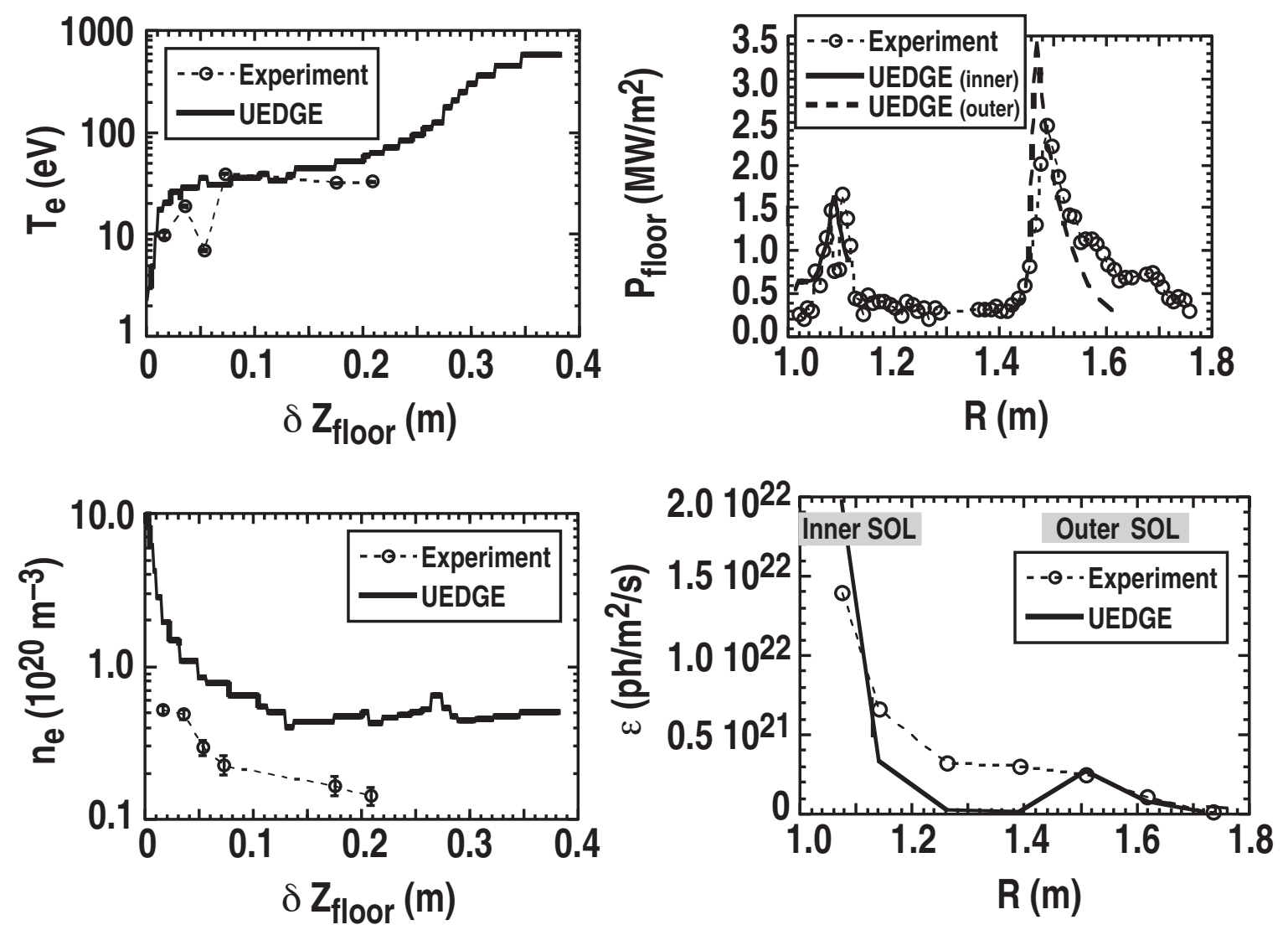

G.D. Porter Figure 5 
(a)

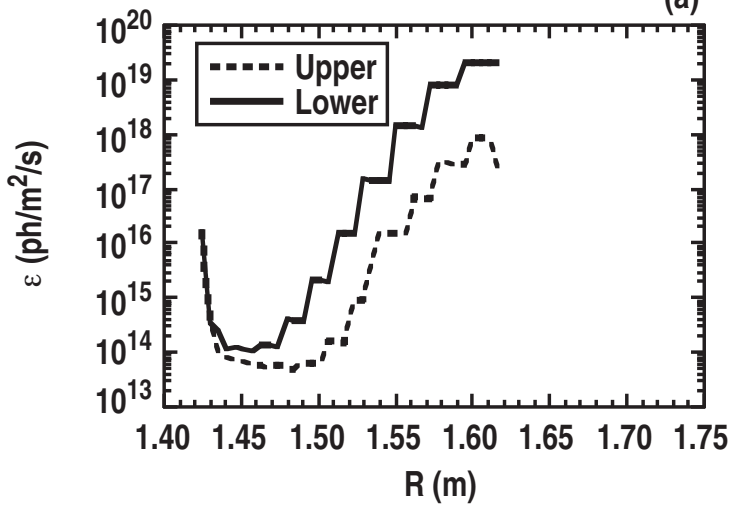

(b)

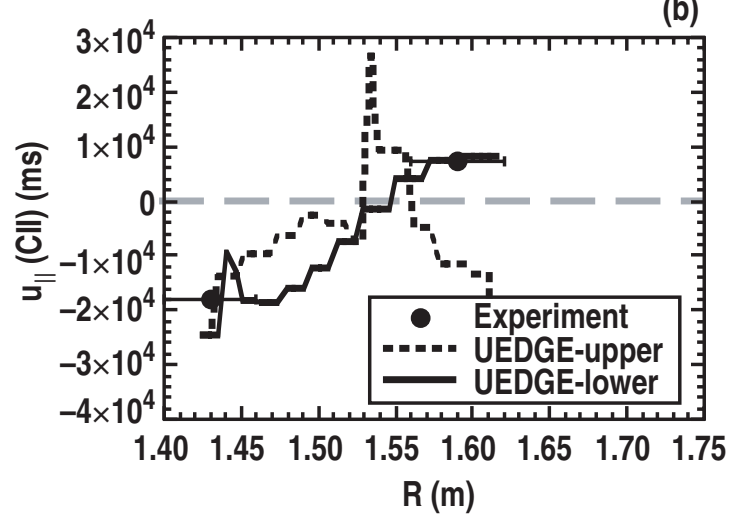

(c)

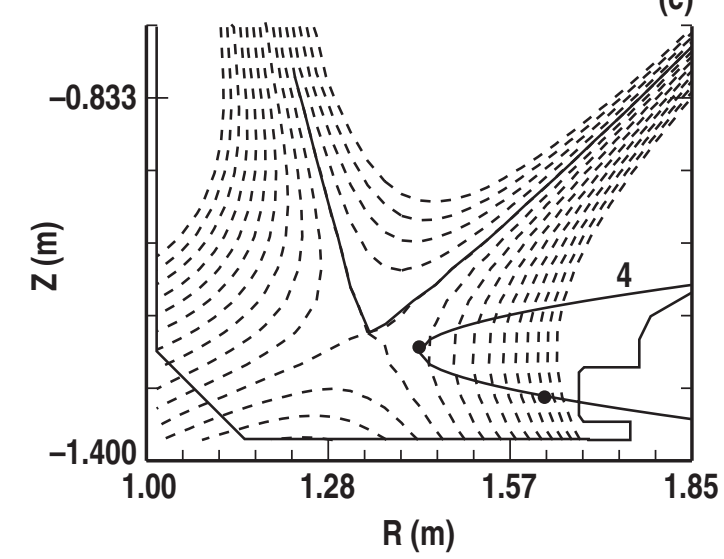

G.D. Porter Figure 6 


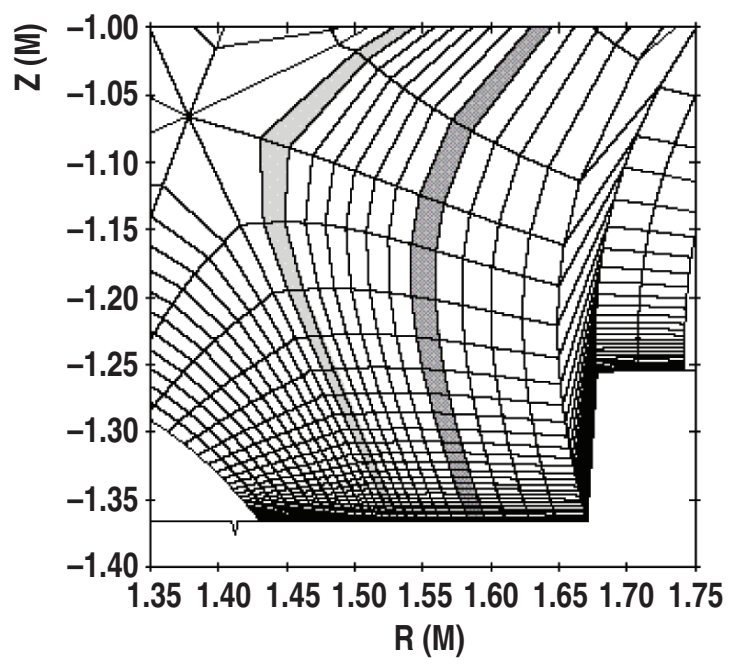

G.D. Porter Figure 7 

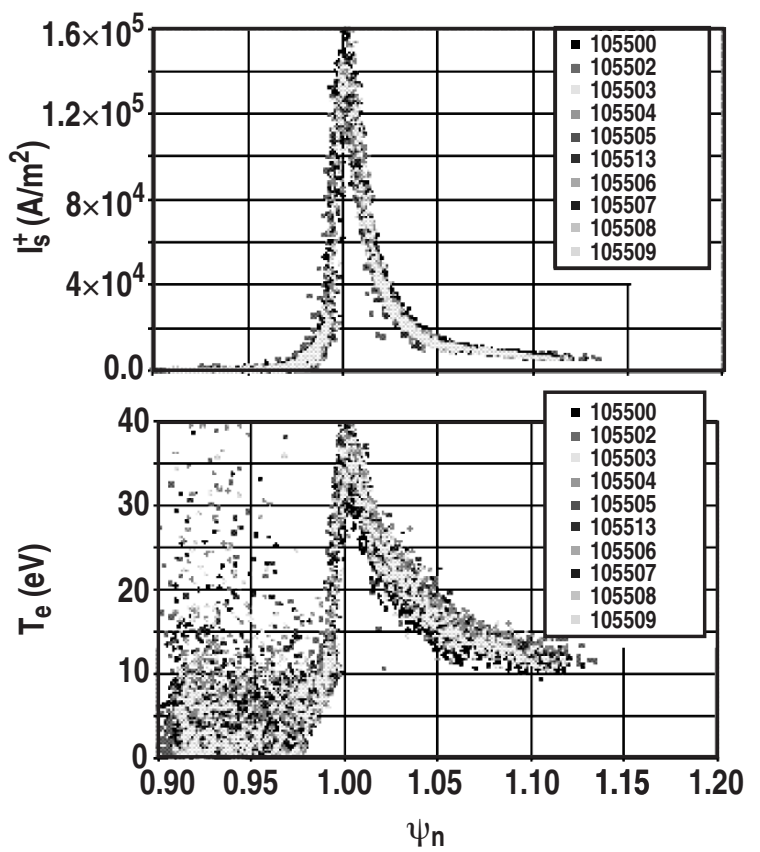

G.D. Porter Figure 8 

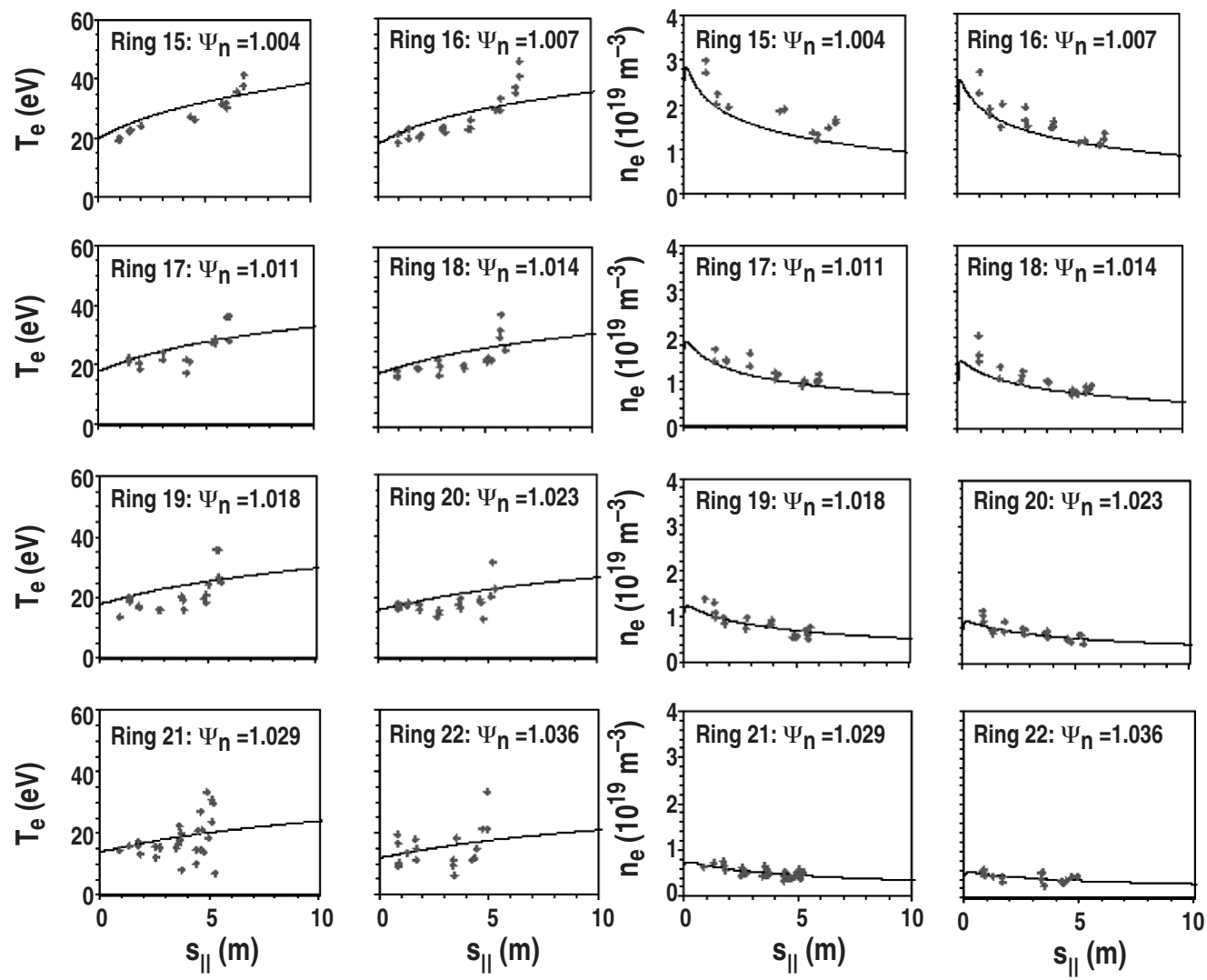

\section{G.D. Porter Figure 9}



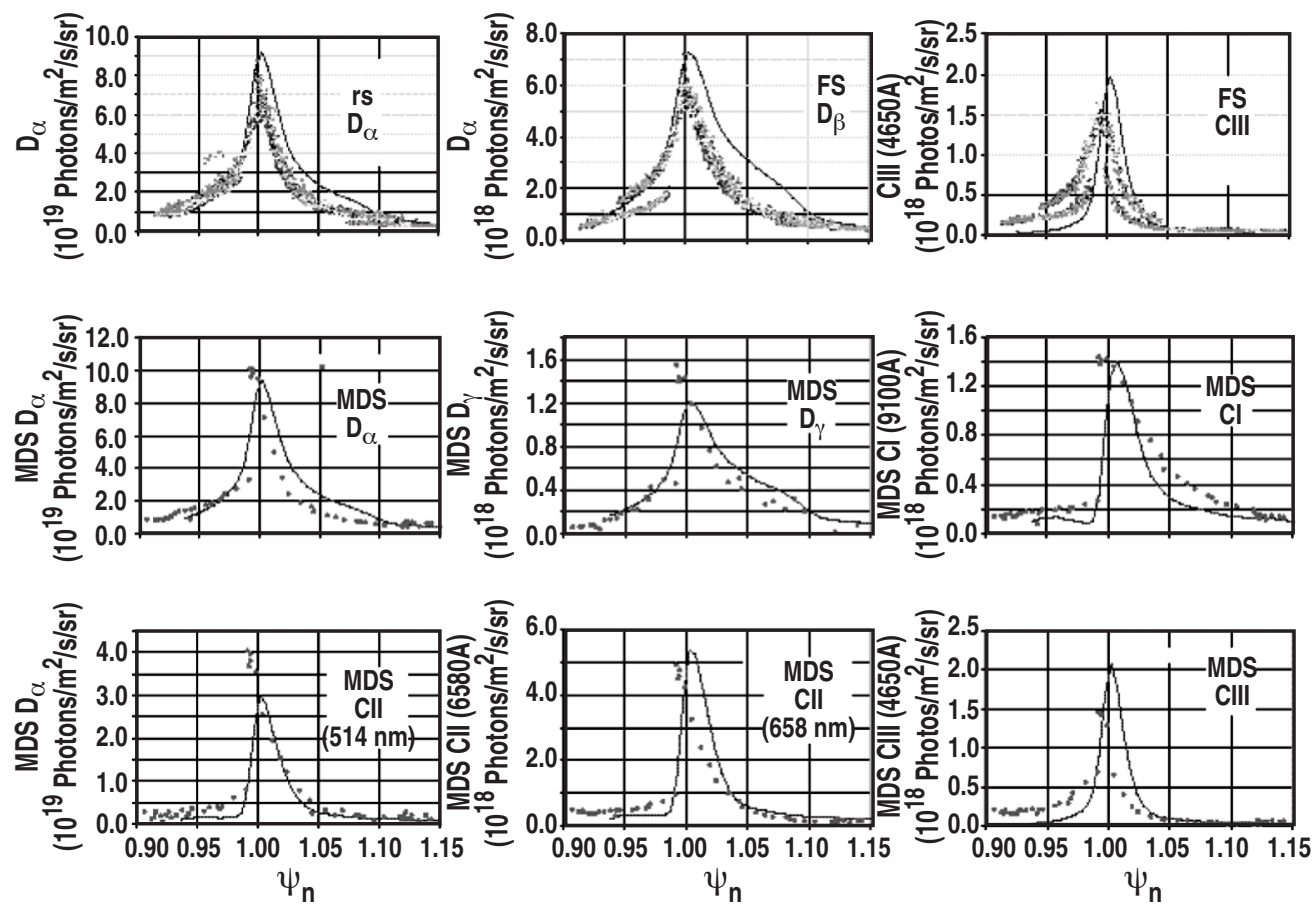

G.D. Porter Figure 10 


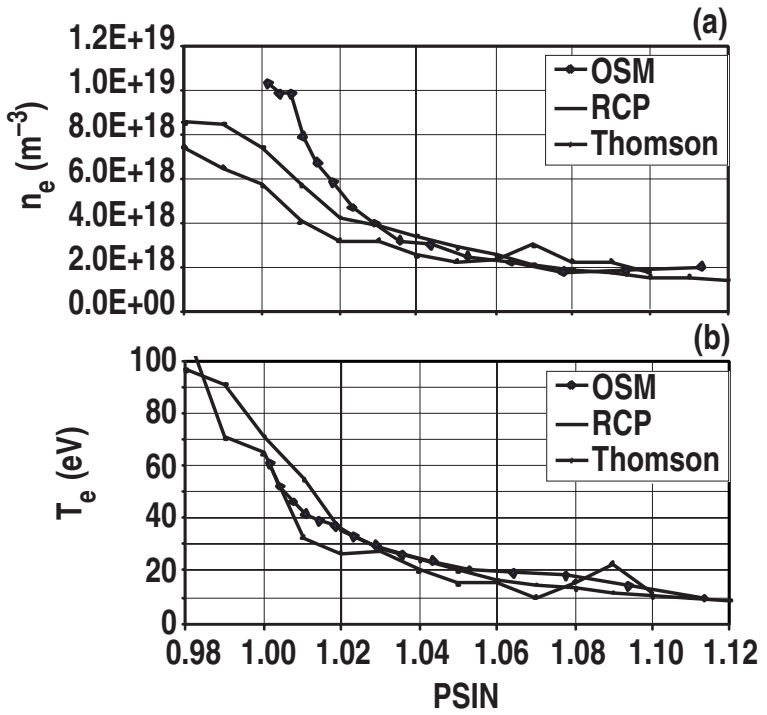

G.D. Porter Figure 11 